\title{
Low $C T$ attenuation and high fatty infiltration rate of psoas are risk factors for incisional hernias after appendicectomy: a cross-sectional single-center study from China
}

Xuechao Du ${ }^{1 \dagger}$, Pengtao Sun ${ }^{2 \dagger}$, Yuchang Yan ${ }^{1}$, Xiang Gong ${ }^{1}$, Yufei Lian ${ }^{1}$ and Zhenyu Pan ${ }^{1 *}$ (D

\begin{abstract}
Background: Decreased computed tomography (CT) attenuation of muscle is independently associated with muscle weakness. The CT attenuation of the abdominal wall muscles may correlate with that of the psoas in patients without ventral hernias. This means that the $\mathrm{CT}$ attenuation of the psoas may be related to the occurrence of incisional hernias $(\mathrm{IH})$. CT-determined sarcopenia was deemed inefficient in predicting the development of $\mathrm{H}$, while limited attention has been paid to the association between muscle fatty infiltration and incidences of $\mathrm{IH}$. In this study, we aim to investigate whether the psoas' CT measurement parameters, including the average CT attenuation, fatty infiltration rate and psoas muscle index, are associated with $\mathrm{H}$.
\end{abstract}

Methods: In this study, adult patients who had undergone an appendicectomy in the past and had then, for any reason, been hospitalised in our hospital from January 2018 to December 2019 were enrolled. The patients were classified into an IH group and a non-IH group. Their psoas' CT attenuation, fatty infiltration rate (FIR) and psoas muscle index (PMI) were measured or calculated. Sarcopenia was defined according to their PMI. Differences between the two groups' indices were then compared. A logistic regression model was applied to assess the effects of psoas' CT measurement parameters on the occurrence of $\mathrm{IH}$.

Results: One hundred twenty patients were included in this study. The psoas' CT attenuation $(p=0.031)$ and PMI $(p=0.042)$ in the $\mathrm{IH}$ group were significantly lower than those in the non- $\mathrm{H}$ group, and $\mathrm{FIR}$ in the $\mathrm{IH}$ group was significantly higher than in the non-IH group $(p<0.001)$. The patients' psoas' $C T$ attenuation, FIR, PMI, age, gender and whether they had a history of smoking, were all significant factors in the univariate logistic regression analysis. After adjusting for confounding factors, a multivariate logistic regression analysis demonstrated that the psoas' $\mathrm{CT}$ attenuation was an independent protective factor $(p=0.042)$, and FIR was an independent risk factor $(p=0.018)$, while neither PMI $(p=0.118)$ nor sarcopenia $(p=0.663)$ showed a significant effect on the incidence of $\mathrm{IH}$.

(Continued on next page)

\footnotetext{
* Correspondence: panzhenyu@ccmu.edu.cn

${ }^{+}$Xuechao Du and Pengtao Sun contributed equally to this work.

'Department of Radiology, Beijing Chaoyang Hospital, Capital Medical University, 5 Jingyuan Road, Shijingshan District, Beijing 100043, China

Full list of author information is available at the end of the article
}

(c) The Author(s). 2021 Open Access This article is licensed under a Creative Commons Attribution 4.0 International License, which permits use, sharing, adaptation, distribution and reproduction in any medium or format, as long as you give appropriate credit to the original author(s) and the source, provide a link to the Creative Commons licence, and indicate if changes were made. The images or other third party material in this article are included in the article's Creative Commons licence, unless indicated otherwise in a credit line to the material. If material is not included in the article's Creative Commons licence and your intended use is not permitted by statutory regulation or exceeds the permitted use, you will need to obtain permission directly from the copyright holder. To view a copy of this licence, visit http://creativecommons.org/licenses/by/4.0/. The Creative Commons Public Domain Dedication waiver (http://creativecommons.org/publicdomain/zero/1.0/) applies to the data made available in this article, unless otherwise stated in a credit line to the data. 
(Continued from previous page)

Conclusions: When an appendectomy has been performed, a decreased CT attenuation and increased FIR of the psoas can be considered risk factors for $\mathbb{H}$.

Keywords: Incisional hernias, Psoas, Computed tomography, Intramuscular adipose, And sarcopenia

\section{Background}

Sarcopenia is considered a patient-specific imaging biomarker able to predict clinical outcomes. Several imaging modalities can be used to assess muscle mass and to diagnose sarcopenia, including dual-energy X-ray absorptiometry (disadvantage: creates inhomogeneous results among different densitometer brands), computed tomography (CT, disadvantage: high radiation exposure), magnetic resonance imaging (MRI, disadvantages: high equipment costs and low availability), and ultrasound (disadvantages: low reproducibility and low accuracy) [1]. CT, which is routinely performed for evaluating abdominal diseases, is optimal for opportunistically assessing sarcopenia without the need of additional examinations or radiation exposure. Besides diagnosing sarcopenia by measuring muscle size in specific districts, CT can also quantitatively measure muscle attenuation and fatty infiltration. All these CT measurement parameters can reflect muscle degeneration.

Muscle weakness in the abdominal wall is one of the main factors that causes ventral incisional hernias (IH). A decreased CT attenuation of muscle is independently associated with muscle weakness [2]. Therefore, the decrease of CT attenuation in the abdominal wall muscles may be a risk factor for $\mathrm{IH}$. When a ventral hernia develops, the abdominal wall is passively unloaded, resulting in atrophic changes in the unloaded skeletal muscles [3]. Since the abdominal wall muscles have a different status before and after the occurrence of a ventral hernia, the $\mathrm{CT}$ attenuation in the abdominal wall muscles of patients with $\mathrm{IH}$ cannot accurately reflect the status of abdominal wall muscles before the occurrence of a hernia. The CT attenuation of the abdominal wall muscles may correlate with that of the psoas in patients that do not have a ventral hernia [4]. Therefore, we hypothesise that the $\mathrm{CT}$ attenuation of the psoas is related to the occurrence of IH. CT-determined sarcopenia has been shown to be an independent and unfavourable predicting factor for various abdominal diseases [5-7]. However, a few studies have concluded that CTdetermined sarcopenia is not sufficient to predict the development of $\mathrm{IH}[8]$. Another indicator, and one that very limited attention has been paid to, is the association between muscle fatty infiltration (another indicator of muscle degeneration) and $\mathrm{IH}$ incidences. In this study therefore, we aim to investigate if the psoas' CT measurement parameters, including average $\mathrm{CT}$ attenuation, fatty infiltration rate (FIR) and psoas muscle index (PMI), are associated with IH. This is explored by measuring the psoas' parameters on the CT images from both $\mathrm{IH}$ and non- $\mathrm{IH}$ patients that have undergone an appendectomy procedure due to appendicitis in the past.

\section{Methods}

Adult patients ( $>18$ years old) who have had an appendicectomy due to appendicitis and were hospitalised in our hospital from January 2018 to December 2019, were consecutively enrolled in this study. The inclusion criterion was: patients had undergone an abdominal CT examination during their hospitalisation. The exclusion criteria were: (a) various factors that could affect muscle mass, including malignant tumours, taking medicines such as glucocorticoids or thyroxine, endocrine diseases, chronic exercise limitations, organ failure and uncontrolled cardiopulmonary diseases; (b) having a lumbosacral transitional anatomy; and (c) having ventral hernias in areas other than appendicectomy incisions. The patients were classified into an IH group (clinically or surgically confirmed incisional hernias at appendicectomy incisions) and a non-IH group. Data on age, gender, height, weight, a history of smoking, hypertension and diabetes were recorded. Body mass index (BMI, kg/ $\mathrm{m}^{2}$ ) was calculated by dividing their weight in kilograms by the square of their height in metres. Our hospital's Research Ethics Review Committee reviewed and approved this study. All methods in this study were carried out in accordance with the Helsinki guidelines and declaration.

\section{CT scan and image measurements}

The CT scans were performed using gemstone CT spectroscopy (Discovery CT 750 HD, GE Healthcare). Imaging protocols included: $120 \mathrm{kVp}$, tube current modulation, pitch 0.984, slice thickness $5 \mathrm{~mm}$, slice spacing $5 \mathrm{~mm}$. Reconstructed images with a $0.625 \mathrm{~mm}$ slice thickness were obtained for further analysis. CT measurements were independently carried out by two experienced radiologists. At the GE AW 4.6 workstation, bilateral psoas muscles were measured separately at the level of the third lumbar vertebra (L3), upon which both the transverse processes were visible, as previously described [9]. By manually outlining both the left and the right psoas, the cross-sectional area $\left(\mathrm{cm}^{2}\right)$ and CT attenuation were automatically calculated. The intramuscular adipose tissue area in the psoas 
was automatically calculated using the threshold between $-190 \mathrm{HU}$ to $-30 \mathrm{HU}$. The FIR (\%) was obtained by dividing the fatty area of the bilateral psoas by the total area of the bilateral psoas. PMI (bilateral psoas cross-sectional area adjusted to a patient's height squared, $\mathrm{cm}^{2} / \mathrm{m}^{2}$ ), the mean bilateral CT attenuation and FIR were calculated. According to previous research carried out on Asian patients [10], a male PMI of $<5.923 \mathrm{~cm}^{2} / \mathrm{m}^{2}$ or a female PMI of $<3.999 \mathrm{~cm}^{2} / \mathrm{m}^{2}$ was defined as sarcopenia.

\section{Statistical analysis}

Quantitative data (such as CT attenuation, FIR, PMI, age, and BMI) were either represented as mean \pm standard deviation, or as the median with an interquartile range. Qualitative data (such as gender, smoking or nonsmoking, hypertension, and diabetes) were reported as counts and percentages. Statistical analyses were performed using SAS version 9.1 (SAS, Cary, NC, USA) and SPSS version 26.0 (SPSS Inc.). The intraclass correlation coefficient (ICC) was used to evaluate the repeatability of the psoas' CT measurements. Quantitative variables were compared between the two groups by using an independent sample t-test or the Wilcoxon signed rank test. Qualitative variables were compared using the chisquare test. A logistic regression model was created to assess the potential influence of the psoas' measurement parameters on having a risk of $\mathrm{IH}$. The MedCalc software was used for receiver operating characteristic curve analysis to obtain the cut-off values for both the CT attenuation and FIR for IH. Statistical significance was defined at a $p<0.05$ (2-sided).

\section{Results}

One hundred twenty consecutive patients were included in this study. Their demographic and clinical characteristics are summarised in Table 1.

\section{Repeatability of CT measurements}

The results show that the inter-rater ICC of the psoas' CT attenuation, muscle area, and FIR are 0.959, 0.996, and $0.914(P<0.001)$, respectively. It is suggested that the CT results' repeatability is good, and that the analysis results in this article are credible.

\section{Comparison of demographic data and CT measurements between IH and non-IH groups (Table 2)}

The psoas' $C T$ attenuation $(\mathrm{t}=-2.18, p=0.031)$ and PMI $(\mathrm{Z}=-2.03, p=0.042)$ in the IH group were significantly lower than those in the non-IH group, and the FIR in IH patients was statistically higher than in non-IH patients $(\mathrm{Z}=3.57, p<0.001)$. IH patients were, on average, 5 years older than non-IH patients $(\mathrm{t}=2.26, p=0.026)$. In addition, in the IH group, more than half were women (63.3\%), while by contrast, females only accounted for
Table 1 Demographic and clinical characteristics of the included patients

\begin{tabular}{ll}
\hline Patient data & $(\boldsymbol{n}=\mathbf{1 2 0})$ \\
\hline Age $(\mathrm{y})$ & $62.5 \pm 13.3$ \\
Gender $(\mathrm{n}, \%)$ & \\
Male & $55(45.8 \%)$ \\
Female & $65(54.2 \%)$ \\
$\mathrm{BMI}^{\mathrm{a}}\left(\mathrm{kg} / \mathrm{m}^{2}\right)$ & $25.9(23.8-27.7)$ \\
$<18.5$, underweight $(\mathrm{n}, \%)$ & $0(0 \%)$ \\
18.5-25.0, normal weight $(\mathrm{n}, \%)$ & $49(40.8 \%)$ \\
$25.0-30.0$, overweight $(\mathrm{n}, \%)$ & $58(48.3 \%)$ \\
$>30$, obesity $(\mathrm{n}, \%)$ & $12(10 \%)$ \\
Smoking $(\mathrm{n}, \%)$ & \\
Yes & $84(70.0 \%)$ \\
No & $36(30.0 \%)$ \\
Hypertension $(\mathrm{n}, \%)$ & \\
Yes & $58(48.3 \%)$ \\
No & $62(51.7 \%)$ \\
Diabetes $(\mathrm{n}, \%)$ & \\
Yes & $23(19.2 \%)$ \\
No & $9780.8 \%)$ \\
\hline
\end{tabular}

$B M I$ Body mass index

${ }^{a}$ One patient's BMI is missing as he was too weak to stand and have his weight measurement taken

$45.0 \%$ in the non-IH group $\left(\chi^{2}=4.06, p=0.044\right)$. The proportion of smokers in the $\mathrm{IH}$ group meanwhile was 1.4 times that of those in the non-IH group $(\mathrm{X} 2=7.78, p=$ 0.005). However, BMI, diabetes and hypertension were comparable between the two groups $(p>0.05)$.

\section{Logistic regression analysis of risk factors of $\mathrm{IH}$}

The univariate logistic regression analysis (Table 3) shows that the psoas' CT attenuation, FIR, PMI, age, gender and a history of smoking were all factors that were associated with $\mathrm{IH}$. After adjusting for age, gender and a history of smoking in multivariate logistic regression analysis, CT attenuation was deemed an independent protective factor (OR 0.94, 95\% CI 0.88-0.99, $p$ $=0.042)$, and FIR an independent risk factor (OR 1.34, $95 \%$ CI 1.05-1.70, $p=0.018$ ). By contrast, PMI (OR 0.78, 95\% CI 0.56-1.07, $p=0.118$ ) and sarcopenia (OR 0.84, 95\% CI 0.38-1.87, $p=0.663$ ) showed no effect on IH (Table 4) (Fig. 1).

As shown in Fig. 2, the area under the curves of the CT attenuation and FIR are 0.604 (95\% CI 0.51-0.69), and 0.689 (95\% CI $0.60-0.77$ ), respectively. FIR had a significantly higher diagnostic efficacy than the CT attenuation $(\mathrm{Z}=2.610, p=0.009)$. The cut-off value for FIR was $3.8 \%$, with a sensitivity of $58.33 \%$ and a specificity of $73.33 \%$. The cut-off value for CT attenuation, was $43.5 \mathrm{HU}$, with a sensitivity of $66.67 \%$ and a specificity of $51.67 \%$. 
Table 2 Comparison of demographic data and CT measurements between $\mathrm{H}$ and non- $\mathrm{H}$ groups

\begin{tabular}{|c|c|c|c|c|}
\hline & $\mathrm{IH}(n=60)$ & non-IH $(n=60)$ & Statistic & $P$ value \\
\hline $\mathrm{CT}$ attenuation (HU) & $40.2 \pm 7.6$ & $43.0 \pm 6.8$ & -2.180 & $0.031^{a}$ \\
\hline FIR (\%) & $4.3(3.1-5.5)$ & $3.1(2.0-4.2)$ & 3.567 & $<0.001^{b}$ \\
\hline PMI $\left(\mathrm{cm}^{2} / \mathrm{m}^{2}\right)$ & $5.0(4.1-5.9)$ & $5.9 \pm 1.9$ & -2.034 & $0.042^{b}$ \\
\hline Age(y) & $65.2 \pm 11.9$ & $59.8 \pm 14.1$ & 2.260 & $0.026^{\mathrm{a}}$ \\
\hline Male (\%) & 36.70 & 55.00 & 4.062 & $0.044^{c}$ \\
\hline $\mathrm{BMI}^{\mathrm{d}}\left(\mathrm{kg} / \mathrm{m}^{2}\right)$ & $26.1(23.9-27.8)$ & $25.6(23.4-27.5)$ & -0.877 & $0.381^{b}$ \\
\hline Smoking (\%) & 81.70 & 58.30 & 7.778 & $0.005^{c}$ \\
\hline Hypertension (\%) & 50.00 & 46.67 & 0.134 & $0.715^{c}$ \\
\hline Diabetes (\%) & 18.30 & 20.00 & 0.054 & $0.817^{c}$ \\
\hline
\end{tabular}

aindependent sample t-test; ${ }^{b}$ Wilcoxon signed rank test; ${ }^{c}$ chi-square test

${ }^{\mathrm{d} O n e}$ patient's BMI is missing as he was too weak to stand and have his weight measurement taken

$I H$ incisional hernias, CT computed tomography, FIR fatty infiltration rate, PMI psoas muscle index; BMI body mass index

\section{Discussion}

In this cross-sectional study, we recruited patients who had undergone an appendicectomy due to appendicitis, and we divided them into two groups based on whether they had developed an IH or not. We explored the relationship between the psoas' CT measurements and IH. After adjusting for age, gender and whether the participants were smoking or non-smoking, the psoas' CT attenuation was deemed a protective factor for $\mathrm{IH}$, and FIR was deemed a risk factor. In addition, it was also noted that PMI and sarcopenia hardly had an effect on the occurrence of $\mathrm{IH}$ at all.Psoas atrophy is mainly manifested by volume reduction and morphological changes, with an unobvious deposition of fat. Therefore, previous studies on IH have mainly focused on the psoas' muscle area and skeletal muscle index. However, in addition to volume reduction, muscle atrophy may pathologically exhibit a fatty infiltration and muscle fibre loss $[3,11]$. Some authors believe that CT attenuation may accurately reflect the number of muscle fibres and the degree of fat deposition [12]. Muscle CT attenuation is related

Table 3 Univariate logistic regression analysis

\begin{tabular}{llll}
\hline & OR & $\mathbf{9 5 \% ~ C l}$ & $\boldsymbol{P}$ value \\
\hline CT attenuation & 0.95 & $0.90-0.99$ & 0.034 \\
FIR & 1.35 & $1.10-1.66$ & 0.004 \\
PMI & 0.76 & $0.60-0.96$ & 0.021 \\
Sarcopenia & 0.80 & $0.37-1.71$ & 0.559 \\
Age & 1.03 & $1.00-1.06$ & 0.028 \\
Gender & 2.11 & $1.02-4.39$ & 0.045 \\
BMI & 1.00 & $0.91-1.10$ & 0.972 \\
Smoking & 3.18 & $1.39-7.30$ & 0.006 \\
Hypertension & 1.14 & $0.56-2.34$ & 0.715 \\
Diabetes & 0.90 & $0.36-2.23$ & 0.817 \\
\hline
\end{tabular}

CT Computed tomography, FIR Fatty infiltration rate, PMI Psoas muscle index, $B M I$ Body mass index to various diseases: paraspinal muscle density is associated with facet joint osteoarthritis, spondylolisthesis and disc narrowing at the same level [13]. Lower thigh muscle CT attenuation could increase the risk of hip fracture, and a standard deviation decrease of just 1 in the thigh muscle $\mathrm{HU}$ value conferred a nearly $40 \%$ increase in the risk of hip fracture [14]. For critically ill adult patients being treated with mechanical ventilation systems, those with a lower skeletal muscle CT value at admission had a higher 6-month mortality rate, and a 10 $\mathrm{HU}$ increase in muscle density was associated with a $14 \%$ decrease in hospital lengths of stay [15]. Furthermore, cancer patients with cachexia and low muscle $\mathrm{CT}$ values had a poor prognosis [16].

Table 4 Multivariate logistic regression analysis

\begin{tabular}{|c|c|c|c|c|}
\hline & & OR & $95 \% \mathrm{Cl}$ & $P$ value \\
\hline \multirow[t]{4}{*}{ CT attenuation } & & 0.94 & $0.88-0.99$ & 0.042 \\
\hline & Age & 0.75 & $0.24-2.32$ & 0.614 \\
\hline & Gender & 0.96 & $0.38-2.45$ & 0.928 \\
\hline & Smoking & 3.31 & $1.19-9.22$ & 0.022 \\
\hline \multirow[t]{4}{*}{ FIR } & & 1.34 & $1.05-1.70$ & 0.018 \\
\hline & Age & 0.77 & $0.25-2.34$ & 0.647 \\
\hline & Gender & 0.85 & $0.32-2.21$ & 0.732 \\
\hline & Smoking & 2.75 & $1.00-7.53$ & 0.049 \\
\hline \multirow[t]{4}{*}{ PMI } & & 0.78 & $0.56-1.07$ & 0.118 \\
\hline & Age & 1.07 & $0.38-2.98$ & 0.897 \\
\hline & Gender & 0.71 & $0.23-2.22$ & 0.556 \\
\hline & Smoking & 3.06 & $1.11-8.44$ & 0.031 \\
\hline \multirow[t]{4}{*}{ Sarcopenia } & & 0.84 & $0.38-1.87$ & 0.663 \\
\hline & Age & 1.29 & $0.47-3.56$ & 0.621 \\
\hline & Gender & 1.26 & $0.52-3.07$ & 0.612 \\
\hline & Smoking & 2.73 & $1.01-7.39$ & 0.047 \\
\hline
\end{tabular}

CT Computed tomography, FIR Fatty infiltration rate, PMI Psoas muscle index 

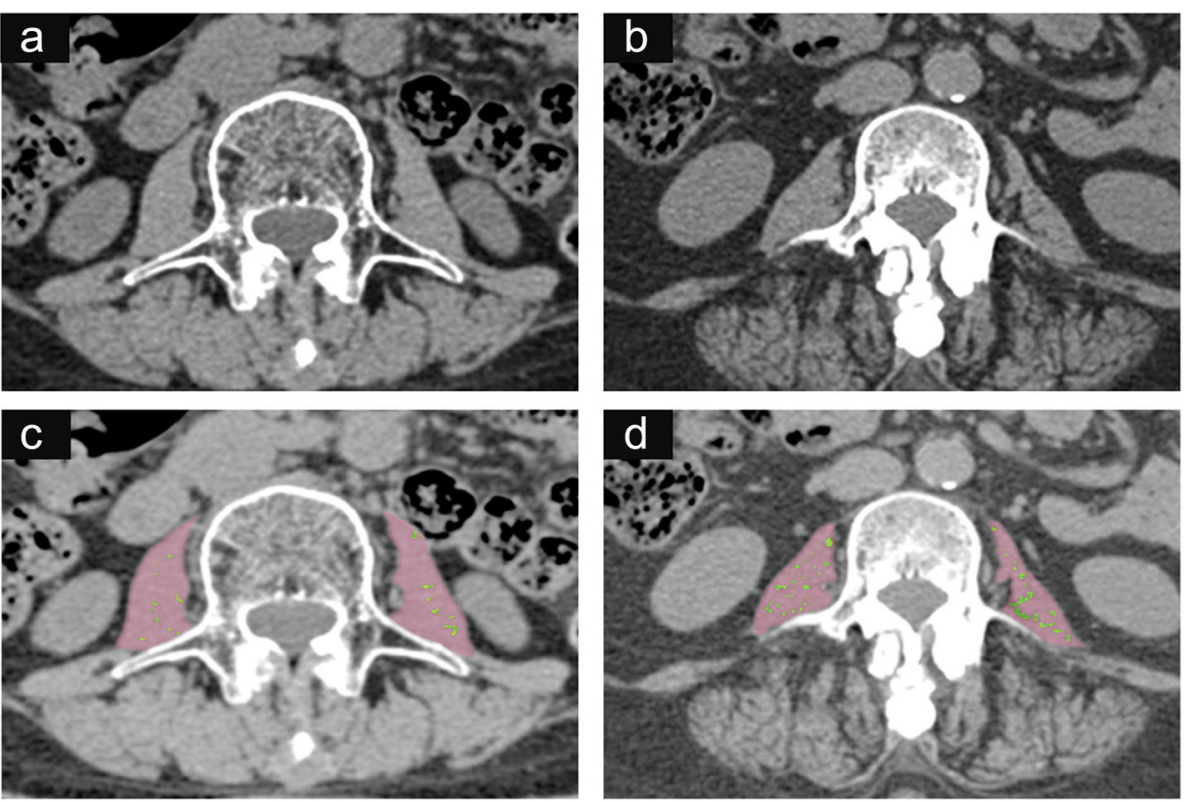

Fig. 1 Measurement of the psoas. Images ( $\mathbf{a}$ and $\mathbf{c}$ ) are from a non-incisional hernia patient (64-year-old, female) and display a higher CT attenuation (47 HU) and a lower fatty infiltration rate (green, 2.1\%); images (b and d) are from an incisional hernia patient (67-year-old, female) and exhibit a lower $\mathrm{CT}$ attenuation $(24 \mathrm{HU}$ ) and a higher fatty infiltration rate (green, 9.4\%). Their psoas muscle indexes are roughly equal (4.0 $\mathrm{cm}^{2} / \mathrm{m}^{2}$ vs. $4.1 \mathrm{~cm}^{2} / \mathrm{m}^{2}$ )

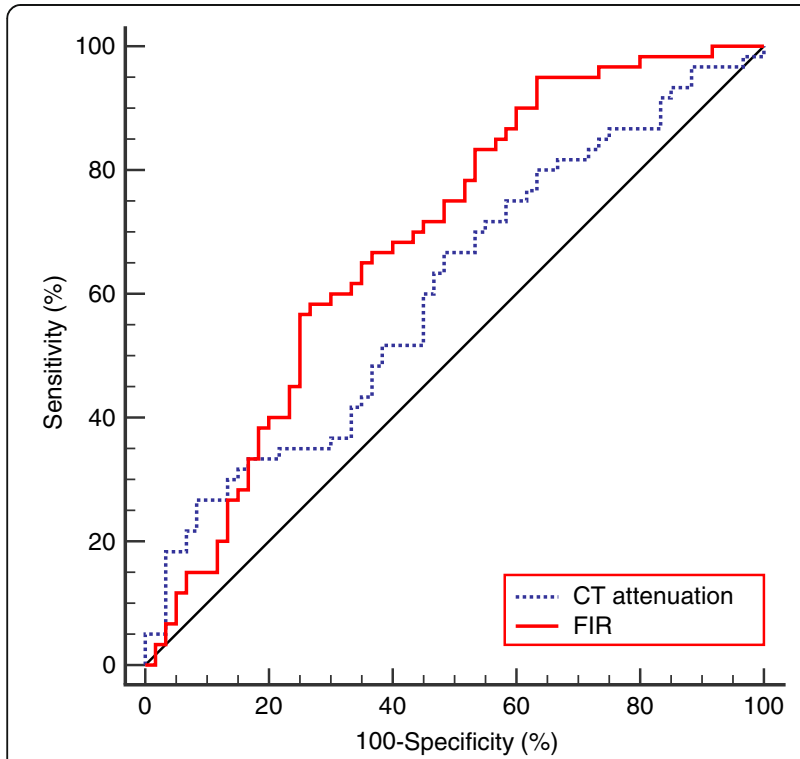

Fig. 2 The receiver operating characteristic curves for $C T$ attenuation and fatty infiltration rate. The fatty infiltration rate has a higher diagnostic value than CT attenuation. The area under the curve of the fatty infiltration rate for diagnosing incisional hernia is 0.689 (95\% Cl $0.60-0.77$ ), with a cut-off at $3.8 \%$, sensitivity of $58.33 \%$, and specificity of $73.3 \%$. The area under the curve of the CT attenuation for diagnosing incisional hernia is 0.604 (95\% Cl 0.51-0.69), with a cut-off at $43.5 \mathrm{HU}$, sensitivity of $66.67 \%$ and specificity of $51.67 \%$
Skeletal muscle density inversely correlated with the length of hospitalisation when following complex abdominal wall hernia surgery [17]. In our study, patients with a lower psoas CT attenuation were more likely to develop IH. It could be that the CT attenuation of the psoas correlates with that of the abdominal wall muscles [4]. A decreased CT attenuation of the muscles is independently associated with muscle weakness [2]. Therefore, a lower CT attenuation of the psoas indirectly reflects a weakness of the abdominal wall muscles, which are susceptible to hernias [18].

In our study, patients with a higher FIR of the psoas were prone to develop IH. Fatty infiltration in skeletal muscle has been identified as a possible cause for loss of muscle quality [2]. Fatty infiltration induces insulin resistance, which impairs the normal capacities for protein synthesis, and subsequently contributes to muscle atrophy $[19,20]$. In previous studies, MRI has often been applied to quantitatively measure the muscle's fat content $[21,22]$, and they concluded that paraspinal fatty infiltration, rather than the muscle's cross-sectional area, was associated with high-intensity pain/disability and structural abnormalities in the lumbar spine [23]. Moreover, in patients with L4-5 single-segment degenerative lumbar spinal stenosis, a fatty infiltration in the multifidus muscles at L5-S1 could be correlated with the disc bulge at the stenosis segment and the reduction of lumbar lordosis [24]. Since MRI examinations are not routinely performed on hernia patients, the MRI-measured muscle 
fat content in these patients is not widely taken into consideration. Previous published research on CT imaging mostly used $\mathrm{HU}$ value to indirectly represent muscle fat content. However, since muscle CT attenuation can be affected by previous surgeries and the deposition of high-density substances, such as calcium and bleeding, it may not effectively reflect the muscle's fat content. Wang C et al. reported that the psoas' CT attenuation in patients with osteoporosis fractures was unexpectedly higher than those without osteoporosis fractures [25], which may be related to intra-muscular haemorrhages or muscle repair following a fracture. In this case, CT attenuation cannot accurately reflect the muscle's fibre content or the degree of fat accumulation. Although relatively complex, measuring the intramuscular fat area or muscle FIR by defining a CT threshold can more accurately reflect the degree of muscle fatty infiltration. So far, few studies have used CT to evaluate fatty infiltration in the muscle. One study that did, by Peter et al., showed that as shoulder strength increases following a shoulder arthroplasty, the rotator cuff fatty infiltration, measured by CT, decreases [26]. To our knowledge, this is the first study to explore the potential relationship between the FIR of the psoas and IH. We concluded that when compared with $\mathrm{CT}$ attenuation, FIR was more closely related to IH.

Previous studies that have investigated the roles of abdominal muscles in malignancies, mostly measured the muscle area in the L3 or L4 cross-sectional image, including the psoas, erector spinae, quadratus lumborum, transversus abdominus, rectus abdominus, and the internal and external obliques. The corresponding skeletal muscle index might predict the prognosis of various malignancies $[9,27]$. CT-determined sarcopenia, that was determined by measuring the level L3 cross-section muscles area, was not a risk factor for the occurrence of IH [28]; although it could prolong the postoperative hospital stay [17]. It is very labour-intensive to measure all the muscle areas in a cross-sectional image, since it must be performed using a specific post-processing software by defining the range of the CT values to exclude intermuscular fat. To only measure the psoas area at level L3 is simple however, and the corresponding muscle index (PMI) correlates with the whole-body muscle mass [29]. A decrease in PMI indicates a decline in the whole-body muscle mass (including the abdominal wall muscles), which results in a decreased functional capacity. Thus, having a low PMI may constitute a potential risk factor for IH. Although PMI at level L3 was a protective factor for IH in our research's univariate regression analysis, it was not statistically significant in multivariate regression analysis. Sarcopenia meanwhile, as defined by PMI's cut-off values in a previous study (based on an Asian population), was not associated with $\mathrm{IH}$ in neither the univariate nor multivariate regression analyses.
Our study demonstrated that psoas' CT attenuation and FIR were associated with IH, but PMI and sarcopenia were not. PMI and sarcopenia reflect muscle mass, while CT attenuation and FIR are associated with psoas' intramuscular adipose. We therefore speculate that the occurrence of $\mathrm{IH}$ is more related to muscle quality than quantity.

Among our study population, smoking was deemed a significant risk factor for $\mathrm{IH}$, and this is consistent with previous publications $[30,31]$. One hypothesis is that tobacco leads to atheroma, which reduces the blood supply to the abdominal wall. In addition, a decreased collagen deposition in surgical test wounds has been found among smokers [32].

In our study, BMI was not deemed an influencing factor of IH. However, many studies have shown that BMI, especially BMI $>30$ (obesity), is a risk factor for $\mathrm{IH}$ $[33,34]$. Obesity is likely to increase intra-abdominal pressure by putting mechanical stress on the abdominal incision, thus predisposing the occurrence of a hernia. However, the BMI in our population (26.1 in the $\mathrm{IH}$ group, 25.6 in the non-IH group) is lower than in those studies, and only $10.0 \%$ of our patients had a BMI $>30$. Therefore, BMI may not have identified as a risk factor because the majority of our patients were non-obese.

Our univariate analysis identified older age and female sex as significant risk factors for $\mathrm{IH}$, while no significant difference was noted in the multivariate analysis. Different studies report inconsistent results on whether age or gender are risk factors for incisional hernias [35-37]. These discrepancies in the age-specific or sex-specific influences on the risk for IH may reflect differences in the study population and diseases. We also showed two non-significant trends in the multivariate analysis: hypertension and diabetes, both of which are consistent with previous research $[35,36]$.

This study has several limitations. First, wound closure techniques and wound infection are recognised risk factors for $\mathrm{IH}$. However, some patients could not recall the relevant information, since more than 10 years or even decades had passed since their appendectomy to this admission. Taking recall-bias into consideration, wound closure techniques and wound infection were not included in the statistical analysis. Second, the area under the curves of CT attenuation and FIR were not very high in our study. The purpose of our study was to screen the influencing factors of $\mathrm{IH}$ after appendectomy, and to provide a basis for further research. A perfect IH prediction model will be constructed in our follow-up research. Third, the cross-sectional retrospective study design limits our ability to ascertain causality and may cause the inevitability of selection bias. Thus, our conclusion needs to be verified using a larger sample size. 


\section{Conclusions}

In summary, our study indicates that following an appendectomy, a decreased CT attenuation and increased FIR of the psoas are risk factors for IH. For patients who have had an appendectomy, when there is a low psoas CT attenuation or high psoas FIR, a prophylaxis of the hernia should be conducted to reduce the possibility of the occurrence of $\mathrm{IH}$.

\section{Abbreviations}

BMI: Body mass index; CT: Computed tomography; FIR: Fatty infiltration rate; $\mathrm{HU}$ : Hounsfield unit; $॥ \mathrm{H}$ : Incisional hernias; MRI: Magnetic resonance imaging; PMI: Psoas muscle index

\section{Acknowledgements}

Not applicable.

\section{Authors' contributions}

Study concept and design: Xuechao Du, Pengtao Sun, Yuchang Yan, Zhenyu Pan. Clinical Data collection: Xuechao Du, Xiang Gong, Yufei Lian. Image Analysis and interpretation: Xuechao Du, Pengtao Sun, Yuchang Yan. Statistical analysis: Pengtao Sun, Yuchang Yan. Drafting the manuscript: Xuechao Du, Pengtao Sun. Critical revision of the manuscript: Yuchang Yan, Zhenyu Pan, Xiang Gong, Yufei Lian. All authors read and approved the final manuscript.

\section{Funding}

This research did not receive any specific grant from funding agencies in the public, commercial, or not-for-profit sectors.

\section{Availability of data and materials}

The data sets generated during and analysed during the current study are not available due to hospital regulation, but are available from the corresponding author on reasonable request.

\section{Declarations}

Ethics approval and consent to participate

This study was approved by the institutional review board of Beijing Chaoyang Hospital, Capital Medical University. Written informed consent was obtained from each participant.

\section{Consent for publication}

Not applicable.

\section{Competing interests}

The authors declare that they have no competing interests.

\section{Author details}

'Department of Radiology, Beijing Chaoyang Hospital, Capital Medical University, 5 Jingyuan Road, Shijingshan District, Beijing 100043, China. ${ }^{2}$ Department of Radiology, Beijing Shijitan Hospital, Capital Medical University, Beijing 100038, China.

Received: 9 January 2021 Accepted: 23 April 2021

Published online: 30 June 2021

\section{References}

1. Albano D, Messina C, Vitale J, Sconfienza LM. Imaging of sarcopenia: old evidence and new insights. Eur Radiol. 2020;30(4):2199-208.

2. Goodpaster BH, Carlson CL, Visser M, Kelley DE, Scherzinger A, Harris TB, et al. Attenuation of skeletal muscle and strength in the elderly: the health ABC study. J Appl Physiol. 2001;90(6):2157-65.

3. DuBay DA, Choi W, Urbanchek MG, Wang X, Adamson B, Dennis RG, et al. Incisional herniation induces decreased Abdominal Wall compliance via oblique muscle atrophy and fibrosis. Ann Surg. 2007:245(1):140-6.

4. Sebro R, O'Brien L, Torriani M, Bredella MA. Assessment of trunk muscle density using $C T$ and its association with degenerative disc and facet joint disease of the lumbar spine. Skelet Radiol. 2016;45(9):1221-6.
5. Jang M, Park HW, Huh J, Lee JH, Jeong YK, Nah YW, et al. Predictive value of sarcopenia and visceral obesity for postoperative pancreatic fistula after pancreaticoduodenectomy analyzed on clinically acquired CT and MRI. Eur Radiol. 2019:29(5):2417-25.

6. Hamidi M, Ho C, Zeeshan M, O'Keeffe T, Hamza A, Kulvatunyou N, et al. Can sarcopenia quantified by computed tomography scan predict adverse outcomes in emergency general surgery? J Surg Res. 2019;235:141-7.

7. Xie H, Gong Y, Kuang J, Yan L, Ruan G, Tang S, et al. Computed tomography-determined sarcopenia is a useful imaging biomarker for predicting postoperative outcomes in elderly colorectal cancer patients. Cancer Res Treat. 2020:52(3):957-72.

8. Schlosser KA, Maloney SR, Thielan ON, Prasad T, Kercher KW, Augenstein VA et al. Sarcopenia in patients undergoing open ventral hernia repair. Am Surg. 2019;85(9):985-91.

9. van Vledder MG, Levolger S, Ayez N, Verhoef C, Tran TC, ljzermans JN. Body composition and outcome in patients undergoing resection of colorectal liver metastases. Br J Surg. 2012;99(4):550-7.

10. Kim JS, Kim WY, Park HK, Kim MC, Jung W, Ko BS. Simple age specific cutoff value for sarcopenia evaluated by computed tomography. Ann Nutr Metab. 2017;71(3-4):157-63.

11. Amato G, Agrusa A, Romano G, Salamone G, Gulotta G, Silvestri F, et al. Muscle degeneration in inguinal hernia specimens. Hernia. 2012; 16(3):327-31

12. Goodpaster BH, Kelley DE, Thaete FL, He J, Ross R. Skeletal muscle attenuation determined by computed tomography is associated with skeletal muscle lipid content. J Appl Physiol. 2000;89(1):104-10.

13. Kalichman L, Hodges P, Li L, Guermazi A, Hunter DJ. Changes in paraspinal muscles and their association with low back pain and spinal degeneration: CT study. Eur Spine J. 2010;19(7):1136-44.

14. Lang T, Cauley JA, Tylavsky F, Bauer D, Cummings S, Harris TB. Computed tomographic measurements of thigh muscle cross-sectional area and attenuation coefficient predict hip fracture: the health, aging, and body composition study. J Bone Miner Res. 2010;25(3):513-9.

15. Looijaard WG, Dekker IM, Stapel SN, Girbes AR, Twisk JW, Oudemans-van Straaten HM, et al. Skeletal muscle quality as assessed by CT-derived skeletal muscle density is associated with 6-month mortality in mechanically ventilated critically ill patients. Crit Care. 2016;20(1):386.

16. Martin L, Birdsell L, Macdonald N, Reiman T, Clandinin MT, McCargar L, et al. Cancer Cachexia in the age of obesity: skeletal muscle depletion is a powerful prognostic factor, independent of body mass index. J Clin Oncol. 2013:31(12):1539-47.

17. Rinaldi JM, Geletzke AK, Phillips BE, Miller J, Dykes TM, Soybel DI. Sarcopenia and sarcopenic obesity in patients with complex abdominal wall hernias. Am J Surg. 2016;212(5):903-11.

18. Jensen KK, Kjaer M, Jorgensen LN. Isometric abdominal wall muscle strength assessment in individuals with incisional hernia: a prospective reliability study. Hernia. 2016:20(6):831-7.

19. Rivas DA, McDonald DJ, Rice NP, Haran PH, Dolnikowski GG, Fielding RA Diminished anabolic signaling response to insulin induced by intramuscular lipid accumulation is associated with inflammation in aging but not obesity. Am J Physiol Reg I. 2016:310(7):R561-9.

20. Hamrick MW, McGee-Lawrence ME, Frechette DM. Fatty infiltration of skeletal muscle: mechanisms and comparisons with bone marrow adiposity. Front Endocrinol. 2016;7:69.

21. Alizai H, Nardo L, Karampinos DC, Joseph GB, Yap SP, Baum T, et al. Comparison of clinical semi-quantitative assessment of muscle fat infiltration with quantitative assessment using chemical shift-based water/ fat separation in MR studies of the calf of post-menopausal women. Eur Radiol. 2012;22(7):1592-600.

22. Morrow JM, Sinclair CD, Fischmann A, Reilly MM, Hanna MG, Yousry TA, et al. Reproducibility, and age, body-weight and gender dependency of candidate skeletal muscle MRI outcome measures in healthy volunteers. Eur Radiol. 2014;24(7):1610-20

23. Teichtahl AJ, Urquhart DM, Wang Y, Wluka AE, Wijethilake P, O'Sullivan R, et al. Fat infiltration of paraspinal muscles is associated with low back pain, disability, and structural abnormalities in community-based adults. Spine J. 2015;15(7):1593-601

24. Liu Y, Liu Y, Hai Y, Li G, Liu T, Wang Y. Lumbar lordosis reduction and disc bulge may correlate with multifidus muscle fatty infiltration in patients with single-segment degenerative lumbar spinal stenosis. Clin Neurol Neurosurg. 2020;189:105629. 
25. Wang C, Hou X, Zhang Y, Wang L, Cheng XG. Change of the psoas major in postmenopausal spinal osteoporotic fractures: a computed tomography. Chin J Bone Jt. 2016;5(8):577-81. https://doi.org/10.3969/j.issn.2095-252X.201 6.08 .005 .

26. Lapner PLC, Jiang L, Zhang T, Athwal GS. Rotator cuff fatty infiltration and atrophy are associated with functional outcomes in anatomic shoulder arthroplasty. Clin Orthop Relat R. 2015;473(2):674-82.

27. Lieffers JR, Bathe OF, Fassbender K, Winget M, Baracos VE. Sarcopenia is associated with postoperative infection and delayed recovery from colorectal cancer resection surgery. Brit J Cancer. 2012;107(6):931-6.

28. van Rooijen MMJ, Kroese LF, van Vugt JLA, Lange JF. Sarcomania? The inapplicability of sarcopenia measurement in predicting incisional hernia development. World J Surg. 2019;43(3):772-9.

29. Hamaguchi Y, Kaido T, Okumura S, Kobayashi A, Hammad A, Tamai Y, et al. Proposal for new diagnostic criteria for low skeletal muscle mass based on computed tomography imaging in Asian adults. Nutrition. 2016;32(11-12):1200-5.

30. Bewö K, Österberg J, Löfgren M, Sandblom G. Incisional hernias following open gynecological surgery: a population-based study. Arch Gynecol Obstet. 2019;299(5):1313-9.

31. Sørensen LT, Hemmingsen UB, Kirkeby LT, Kallehave F, Jørgensen LN. Smoking is a risk factor for incisional hernia. Arch Surg. 2005;140(2):119-23.

32. Jorgensen LN, Kallehave F, Christensen E, Siana JE, Gottrup F. Less collagen production in smokers. Surgery. 1998;123(4):450-5.

33. Ooms LS, Verhelst J, Jeekel J, ljzermans JN, Lange JF, Terkivatan T. Incidence, risk factors, and treatment of incisional hernia after kidney transplantation: an analysis of 1,564 consecutive patients. Surgery. 2016;159(5):1407-11.

34. Tuttle TM, Huang JL, Kizy S, Altman AM, Nalluri H, Marmor S, et al. Incidence and predictors of incisional hernia after cytoreductive surgery and hyperthermic intraperitoneal chemotherapy. Int J Hyperth. 2019;36(1):812-6.

35. Alhambra-Rodríguez de Guzmán C, Morandeira-Rivas AJ, Herrero-Bogajo ML, Moreno-Sanz C. Incidence and risk factors of incisional hernia after single-incision endoscopic surgery. J Laparoendosc Adv Surg Tech A. 2020;30(3):251-5.

36. Sabajo CR, Olthof PB, Roos D, Dekker JWT. Incisional hernia after laparoscopicassisted right hemicolectomy. World J Surg. 2019;43(12):3172-8.

37. Llaguna OH, Avgerinos DV, Lugo JZ, Matatov T, Abbadessa B, Martz JE, et al. Incidence and risk factors for the development of incisional hernia following elective laparoscopic versus open colon resections. Am J Surg. 2010;200(2):265-9.

\section{Publisher's Note}

Springer Nature remains neutral with regard to jurisdictional claims in published maps and institutional affiliations.

Ready to submit your research? Choose BMC and benefit from:

- fast, convenient online submission

- thorough peer review by experienced researchers in your field

- rapid publication on acceptance

- support for research data, including large and complex data types

- gold Open Access which fosters wider collaboration and increased citations

- maximum visibility for your research: over $100 \mathrm{M}$ website views per year

At $\mathrm{BMC}$, research is always in progress.

Learn more biomedcentral.com/submissions 